\section{Walking Before an Ultrasound Assessment Increases the Enthesis Score Significantly}

\section{To the Editor:}

Inflammatory enthesis involvement is a characteristic feature of spondyloarthropathies $(\mathrm{SpA})$; it is considered the primary lesion in this disease ${ }^{1}$. The MAdrid Sonographic Enthesis Index (MASEI) score was recently validated for SpA diagnostic classification ${ }^{2}$. MASEI scores range from 0 to 136 , and a value $\geq 18$ was established as the best cutoff point to differentiate between cases and healthy controls (sensitivity of $83.3 \%$, specificity $82.8 \%$ ).

Sporting activities such as running and aerobic exercise are known to result in foot and ankle injuries, such as tendon thickening, edema, and bursitis, that could influence ultrasound (US) assessment of enthesis ${ }^{3}$. To our knowledge, the effect of a mild sport activity on an US assessment of enthesis has never been studied. We investigated the influence of a 30-minute walk just before an US assessment of enthesis using the MASEI score.

Healthy subjects with no musculoskeletal disease, kidney disease, or ongoing treatment with nonsteroidal antiinflammatory drugs were invited to participate. Subjects doing more than 1 hour of sports weekly were excluded.

Two US assessments were performed on the same day: the first after a 15-minute rest in the US evaluation room, and the second after a 30-minute treadmill walk (speed $5 \mathrm{~km} / \mathrm{h}$ ). US assessments were performed by an unblinded experienced rheumatologist (YG; intraobserver reliability in US enthesis assessment: kappa $=0.84$ ) using an Antares V4 US unit (Siemens, Malvern, PA, USA) with an 11.4 MHz linear array transducer. Six entheses were investigated bilaterally: proximal plantar fascia, distal Achilles tendon, distal and proximal patellar ligament, distal quadriceps, and brachial triceps tendons. Enthesis thickness, structure, calcifications, erosions, bursae, and power Doppler signal were considered on both longitudinal and transversal planes. The MASEI score was calculated for each subject at the 2 study times (after rest $=$ rest-score and after walk $=$ walk-score)

To test differences between each patient's paired data, we used the Wilcoxon signed-rank test (Statview v. 5 software; SAS Institute, Cary, NC, USA).
Nineteen healthy subjects were recruited (mean age 43.2 years, range 18-60). Mean MASEI rest-score and walk-score were statistically different, 4.89 (range 0-11) and 6.46 (range $0-12$ ), respectively $(\mathrm{p}=0.007$ ). After the walk period, the MASEI score increased in 9 subjects (47\%), with appearance of retrocalcaneal bursitis in 7 (Figure 1), power Doppler signals of the posterior pole of the calcaneus in 2 (Figure 2), infrapatellar bursitis in 4 , and proximal patellar ligament thickening $(>4 \mathrm{~mm})$ in 2 subjects. Our results reinforce the validity of the MASEI score for classification of diagnosis of SpA. Even after the walk phase, none of the healthy subjects presented a MASEI score over 18.

To our knowledge this is the first study to demonstrate that a simple walk exercise significantly increases an US enthesis score in healthy patients. These results may prompt investigators to propose a systematic rest before any US assessment of enthesis.

JEAN-CAMILLE MÉRIC, MD; YAN GRANDGEORGE, MD; GUILLAUME LOTITO, MD; THAO PHAM, MD, Department of Rheumatology, Conception Hospital, AP-HM, Mediterranean University, Aix-Marseille II, Marseille, France. Address correspondence to Prof. T. Pham, Department of Rheumatology, Conception Hospital,

147 Boulevard Baille, 13005 Marseille, France.

E-mail: thao.pham@ap-hm.fr

\section{REFERENCES}

1. McGonagle D, Khan MA, Marzo-Ortega H, O'Connor P, Gibbon W, Emery P. Enthesitis in spondyloarthropathy. Curr Opin Rheumatol 1999;11:244-50.

2. de Miguel E, Cobo T, Munoz-Fernandez S, Naredo E, Uson J, Acebes JC, et al. Validity of enthesis ultrasound assessment in spondyloarthropathy. Ann Rheum Dis 2009;68:169-74.

3. Benjamin M, Toumi H, Ralphs JR, Bydder G, Best TM, Milz S. Where tendons and ligaments meet bone: attachment sites ('entheses') in relation to exercise and/or mechanical load. J Anat 2006;208:471-90

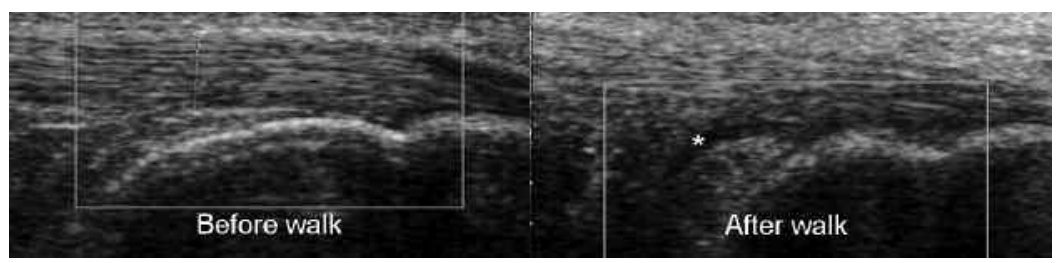

Figure 1. Ultrasound assessment of distal Achilles tendon before and after a 30-minute walk on a treadmill, with appearance of retrocalcaneal bursitis after the walk. *Retrocalcaneal bursitis.

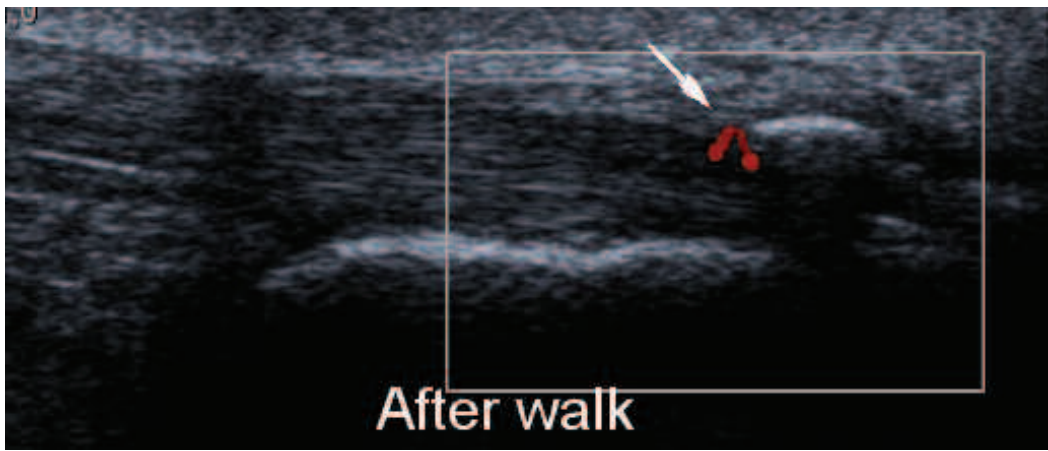

Figure 2. Ultrasound assessment of distal Achilles tendon after a 30-minute walk on a treadmill, with appearance of a power Doppler signal at the posterior pole of the calcaneus (arrow). 